\title{
AN INFINITE PRIMARY ABELIAN GROUP WITHOUT PROPER ISOMORPHIC SUBGROUPS ${ }^{1}$
}

\author{
BY PETER CRAWLEY
}

\author{
Communicated by R. S. Pierce, April 18, 1962
}

In a recent article ${ }^{2}$ Beaumont and Pierce considered the problem of determining those abelian groups which have proper isomorphic subgroups. For primary groups they obtained the following: if the cardinality of a reduced primary abelian group $G$ is either $\boldsymbol{\aleph}_{0}$ or greater than $2 \aleph_{0}$, then $G$ has a proper isomorphic subgroup. The question remained whether or not this result holds for all infinite reduced primary groups. Here we answer this question by showing that there exists an infinite primary abelian group without elements of infinite height which has no proper isomorphic subgroups.

Throughout this note the usual terminology and notation ${ }^{3}$ is employed with possibly the following exception: if $x$ is an element of a group then $[x]$ denotes the cyclic subgroup generated by $x$. The cardinality of a set $M$ is denoted by $|M|$.

Let $p$ be a fixed but arbitrary prime. For each $k=1,2,3, \cdots$ let $C_{k}=\left[g_{k}\right]$ be a cyclic group of order $p^{k}$ generated by the element $g_{k}$. Let $C$ be the torsion subgroup of the complete direct sum of the groups $C_{k}(k=1,2, \cdots)$. The elements $x$ in $C$ are then countable sequences

$$
x=\left(x_{1}, x_{2}, \cdots, x_{k}, \cdots\right)
$$

where $x_{k} \in C_{k}$ and the orders of the $x_{k}$ 's are uniformly bounded; the element $x_{k}$ is called the $k$ th component of $x$. Also $C$ is a closed $p$-group in that every Cauchy sequence in $C$ has a limit. ${ }^{4}$ Set $h_{k}=p^{k-1} g_{k}$, and define the elements $b_{k}$ and $c_{k}(k=1,2, \cdots)$ by

$$
\begin{aligned}
b_{k} & =\left(0, \cdots, 0, g_{k}, 0, \cdots\right), \\
c_{k} & =\left(0, \cdots, 0, h_{k}, 0, \cdots\right)=p^{k-1} b_{k} .
\end{aligned}
$$

Each $c_{k}$ is then an element of order $p$. Moreover if $x$ is an element in $C$ of order $p$, then there is a sequence of integers $u_{1}, u_{2}, u_{8}, \cdots$ such that

${ }^{1}$ This work was supported in part by National Science Foundation grant NSF G-17957.

2 R. A. Beaumont and R. S. Pierce, Partly transitive modules and modules with proper isomorphic submodules, Trans. Amer. Math. Soc. 91 (1959), 209-219.

${ }^{3}$ See, for example, L. Fuchs, Abelian groups, Budapest, 1958.

4 Ibid., p. 114. 


$$
x=\left(u_{1} h_{1}, u_{2} h_{2}, u_{3} h_{3}, \cdots\right),
$$

and $x$ is the limit in $C$ of the Cauchy sequence

$$
u_{1} c_{1}, u_{1} c_{1}+u_{2} c_{2}, u_{1} c_{1}+u_{2} c_{2}+u_{3} c_{3}, \cdots \text {. }
$$

Let $\sigma=\left\{s_{1}, s_{2}, s_{3}, \cdots\right\}$ be a sequence of elements in $C$ of order $p$ such that $s_{k}$ has height at least $k-1$ for each $k=1,2, \cdots$. If $x=\left(u_{1} h_{1}, u_{2} h_{2}, u_{3} h_{3}, \cdots\right)$ is any element in $C$ of order $p$, then the elements

$$
u_{1} s_{1}, u_{1} s_{1}+u_{2} s_{2}, u_{1} s_{1}+u_{2} s_{2}+u_{3} s_{3}, \cdots
$$

form a Cauchy sequence and hence have a limit in $C$; this limit we denote by $x^{\sigma}$. Furthermore, if $\sigma$ has the property, that for no integers $u$ and $v$ is it true that $s_{k}=u c_{k}$ for all $k \geqq v$, then $\sigma$ is called distinguished.

We now observe the following: if $\sigma=\left\{s_{1}, s_{2}, s_{3}, \ldots\right\}$ is distinguished, then there exists a set $T_{\sigma}$ of elements of order $p$ such that $\left|T_{\sigma}\right|=2^{\aleph_{0}}$ and such that for every pair of distinct elements $x, y \in T_{\sigma}$,

$$
[x]+\left[x^{\sigma}\right]+[y]+\left[y^{\sigma}\right]=[x] \oplus\left[x^{\sigma}\right] \oplus[y] \oplus\left[y^{\sigma}\right] .
$$

In constructing $T_{\sigma}$ there are three cases to consider. The first: there is an infinite sequence of integers $n_{1}, n_{2}, n_{3}, \cdots$ such that $s_{n_{k}}$ has height greater than $n_{k}-1$ for each $k=1,2, \cdots$ (and hence each $s_{n_{k}}$ has its first $n_{k}$ components zero). In this case we may assume that $n_{k+1}-1$ is greater than the height of $s_{n_{k}}$ for each $k=1,2, \cdots$. Let $S \subseteq\left\{n_{2}, n_{3}, n_{4}, \cdots\right\}$, and define an element $x(S)$ by

$$
x(S)_{i}= \begin{cases}0 & \text { if } i \neq n_{1} \text { and } i \notin S, \\ h_{i} & \text { if } i=n_{1} \text { or } i \in S,\end{cases}
$$

where $x(S)_{i}$ denotes the $i$ th component of $x(S)$. Then the set $T_{\sigma}=\left\{x(S) \mid S \subseteq\left\{n_{2}, n_{3}, n_{4}, \cdots\right\}\right\}$ is easily seen to have the desired properties. The second case is: there is an infinite sequence of integers $n_{1}, n_{2}, n_{3}, \cdots$ such that $s_{n_{k}}$ has height $n_{k}-1$ and has more than one nonzero component for each $k=1,2, \cdots$. In this case we may assume that $n_{k+1}$ is greater than the index of the second nonzero component of $s_{n_{k}}$ for each $k$. If for every $S \subseteq\left\{n_{2}, n_{3}, n_{4}, \cdots\right\}$ an element $x(S)$ is defined as in the preceding case, then

$$
T_{\sigma}=\left\{x(S) \mid S \subseteq\left\{n_{2}, n_{3}, n_{4}, \cdots\right\}\right\}
$$

again has the desired properties. The remaining case is the following: there is an integer $m$ such that $s_{i}$ has height $i-1$ and exactly one nonzero component for each $i \geqq m$. In this case, since $\sigma$ is distinguished, there are two infinite disjoint sequences of integers $n_{1}, n_{2}$, 
$n_{3}, \cdots$ and $m_{1}, m_{2}, m_{3}, \cdots$, and two integers $u$ and $v$ such that $u \neq v(\bmod p)$ and such that for each $k$ we have $n_{k}, m_{k} \geqq m, s_{n_{k}}=u c_{n_{k}}$, and $s_{m_{k}}=v c_{m_{k}}$. Let $S \subseteq\{2,3,4, \cdots\}$ and define an element $x(S)$ by

$$
x(S)_{i}= \begin{cases}0 & \text { if } i \neq n_{k} \text { and } i \neq m_{k} \text { for all } k \in S \cup\{1\}, \\ h_{i} & \text { if } i=n_{k} \text { or } i=m_{k} \text { for some } k \in S \cup\{1\},\end{cases}
$$

where $x(S)_{i}$ denotes the $i$ th component of $x(S)$. Then the set $T_{\sigma}$ $=\{x(S) \mid S \subseteq\{2,3,4, \cdots\}\}$ has the required properties.

It is clear that there are $2^{\aleph_{0}}$ distinct distinguished sequences. Let $\Omega$ be the first ordinal of cardinality $2^{\aleph_{0}}$, and let

$$
\alpha \leftrightarrow \sigma_{\alpha}
$$

be a one-one correspondence between the distinguished sequences and the ordinals $\alpha<\Omega$. Let $c$ be any element of order $p$ in $C$ which is not in the subgroup generated by the elements $c_{1}, c_{2}, c_{3}, \cdots$.

We now construct a subgroup $P_{\alpha} \subseteq\{x \in C \mid p x=0\}$ for each $\alpha<\Omega$ with the following properties:

(i) $P_{\alpha} \supseteq P_{\beta} \supseteq\left\{c_{1}, c_{2}, c_{3}, \cdots\right\}$ for all $\beta \leqq \alpha$;

(ii) $\left|P_{\alpha}\right| \leqq|\alpha| \aleph_{0}$;

(iii) $c \notin P_{\alpha}$;

(iv) there exists an element $x_{\alpha} \in P_{\alpha}$ such that $c-x_{\alpha}^{\sigma_{\alpha}} \in P_{\alpha}$.

Suppose $P_{\beta}$ has been constructed for each $\beta<\alpha$. Set

$$
P_{\alpha}^{\prime}=\underset{\beta<\alpha}{\bigcup} P_{\beta}+\left[c_{1}\right]+\left[c_{2}\right]+\left[c_{3}\right]+\cdots \cdot
$$

It then follows that $\left|P_{\alpha}^{\prime}+[c]\right| \leqq|\alpha| \aleph_{0}<2^{\aleph_{0}}$, and since there are $2 \aleph^{\aleph_{0}}$ pairwise disjoint subgroups of form

$$
[x] \oplus\left[x^{\sigma_{\alpha}}\right], \quad x \in T_{\sigma_{\alpha}},
$$

there exists an element $x_{\alpha} \in T_{\sigma_{\alpha}}$ such that

$$
\left(\left[x_{\alpha}\right] \oplus\left[x_{\alpha}^{\sigma_{\alpha}}\right]\right) \cap\left(P_{\alpha}^{\prime}+[c]\right)=0 .
$$

If $c \in P_{\alpha}^{\prime}+\left[x_{\alpha}\right]+\left[c-x_{\alpha}^{\sigma_{\alpha}}\right]$, then there are integers $u, v$ and an element $y \in P_{\alpha}^{\prime}$ such that

$$
c=y+u x_{\alpha}+v\left(c-x_{\alpha}^{\sigma_{\alpha}}\right) .
$$

Consequently

$$
u x_{\alpha}-v x_{\alpha}^{\sigma_{\alpha}} \in P_{\alpha}^{\prime}+[c],
$$

whence $u \equiv v \equiv 0(\bmod p)$. But then $c=y \in P_{\alpha}^{\prime}$, an impossibility. Therefore if we set 


$$
P_{\alpha}=P_{\alpha}^{\prime}+\left[x_{\alpha}\right]+\left[c-x_{\alpha}^{\sigma_{\alpha}}\right],
$$

then $c \notin P_{\alpha}$, and it follows that $P_{\alpha}$ satisfies (i)-(iv).

Having constructed the sequence $P_{\alpha}(\alpha<\Omega)$, define the subgroup $P$ by

$$
P=\underset{\alpha<\Omega}{\bigcup} P_{\alpha}
$$

Let $G$ be a pure subgroup of $C$ such that

$$
G \supseteq\left\{b_{1}, b_{2}, b_{3}, \cdots\right\}
$$

and

$$
\{x \in G \mid p x=0\}=P .
$$

Suppose $f$ is an isomorphic mapping of $G$ into itself. Then the sequence $\sigma_{f}$ of the elements

$$
f\left(c_{1}\right), f\left(c_{2}\right), f\left(c_{3}\right), \cdots
$$

is a sequence of elements of order $p$, and clearly each $f\left(c_{k}\right)$ has height at least $k-1$. Suppose $\sigma_{f}$ is not distinguished. Then there are integers $u$ and $v$ such that $f\left(c_{k}\right)=u c_{k}$ for all $k \geqq v$. Let $Q$ be the subgroup of those elements in $P$ which are limits of Cauchy sequences from the subgroup generated by $c_{v}, c_{v+1}, c_{v+2}, \cdots$, that is, $Q$ is the subgroup of $P$ consisting of these elements whose first $v-1$ components are zero. Observe that $f(x)=u x$ for all $x \in Q$, and since $u$ is necessarily relatively prime to $p$, we have $f(Q)=Q$. Let $H$ be the subgroup of $G$ consisting of those elements whose first $v-1$ components are zero. Since $G$ contains the elements $b_{1}, b_{2}, b_{3}, \cdots, H$ is a pure subgroup of G. Moreover

$$
\{x \in H \mid p x=0\}=Q=\{y \in f(H) \mid p y=0\},
$$

and it follows that $f(H)$ is a pure subgroup of $G$. Let $x$ be any element of order $p$ in $G$. Then there are integers $w_{1}, \cdots, w_{v-1}$ and an element $y \in Q$ such that

$$
x=w_{1} c_{1}+\cdots+w_{v-1} c_{v-1}+y,
$$

and since $f(H)$ is pure we infer that $G / f(H)$ has rank $v-1$. Suppose there is an element $z \in G$ such that $x \equiv p^{v-1} z(\bmod f(H))$. Then $z$ has order $p^{v}$ modulo $f(H)$, and since $f(H)$ is pure we may assume that the actual order of $z$ is $p^{v}$. The order of $p^{v-1} z$ is therefore $p$, and clearly the first $v-1$ components of $p^{v-1} z$ are zero. Hence $p^{v-1} z \in Q$, and as $x-p^{v-1} z \in Q$ we conclude that $x \in Q \subseteq f(H)$. Thus every element in $G / f(H)$ of order $p$ has height at most $v-2$, and it follows that 
$G / f(H)$ is finite. Consequently there exists a finite subgroup $F$ such that

$$
G=F \oplus f(H)
$$

Furthermore

$$
f(G)=F^{\prime} \oplus f(H)
$$

where $F^{\prime}=F \cap f(G)$. Since $f(G) \cong G$, it follows that $F \cong F^{\prime}$; whence $F=F^{\prime}$. Hence $f(G)=G$, and $f$ is an automorphism when $\sigma_{f}$ is not distinguished. Suppose then that $\sigma_{f}$ is distinguished. Then there is an ordinal $\alpha<\Omega$ such that $\sigma_{\alpha}=\sigma_{f}$. If the element $x_{\alpha}$ has the form $x_{\alpha}$ $=\left(u_{1} h_{1}, u_{2} h_{2}, u_{3} h_{3}, \cdots\right)$, then $x_{\alpha}$ is the limit in $C$, and hence the limit in $G$ since $G$ is pure, of the Cauchy sequence

$$
u_{1} c_{1}, u c_{1}+u_{2} c_{2}, u_{1} c_{1}+u_{2} c_{2}+u_{3} c_{3}, \cdots,
$$

and consequently $f\left(x_{\alpha}\right)$ is the limit in $f(G)$ (and hence the limit is $C$ ) of the Cauchy sequence

$$
u_{1} f\left(c_{1}\right), u_{1} f\left(c_{1}\right)+u_{2} f\left(c_{2}\right), u_{1} f\left(c_{1}\right)+u_{2} f\left(c_{2}\right)+u_{3} f\left(c_{3}\right), \cdots .
$$

But this limit is $x_{\alpha}^{\sigma_{f}}=x_{\alpha}^{\sigma_{\alpha}}$, whence $f\left(x_{\alpha}\right)=x_{\alpha}^{\sigma_{\alpha}} \in G$. Since $c-x_{\alpha}^{\sigma_{\alpha}} \in G$ it follows that $c \in G$, a contradiction. Therefore $\sigma_{f}$ cannot be distinguished. We conclude that every isomorphic mapping of $G$ into itself is an automorphism, and the proof is complete.

UNIVERSITY OF MinNESOTA 